\title{
Proton Pump Inhibitors and Risk for Recurrent Clostridium Difficile Infection Among Inpatients
}

\author{
Daniel E. Freedberg, MD ${ }^{1}$, Hojjat Salmasian, MD, $\mathrm{MPH}^{2}$, Carol Friedman, $\mathrm{PhD}^{2}$, and Julian \\ A. Abrams, MD, MS ${ }^{1}$ \\ ${ }^{1}$ Division of Digestive and Liver Diseases, Columbia University Medical Center, New York, New \\ York, USA \\ ${ }^{2}$ Department of Biomedical Informatics, Columbia University, New York, New York, USA
}

\section{Abstract}

Objectives-Observational studies suggest that proton pump inhibitors (PPIs) are a risk factor for incident Clostridium difficile infection (CDI). Data also suggest an association between PPIs and recurrent CDI, although large-scale studies focusing solely on hospitalized patients are lacking. We therefore performed a retrospective cohort analysis of inpatients with incident CDI to assess receipt of PPIs as a risk factor for CDI recurrence in this population.

\begin{abstract}
Methods-Using electronic medical records, we identified hospitalized adult patients between December 1, 2009 and June 30, 2012 with incident CDI, defined as a first positive stool test for $C$. difficile toxin $\mathrm{B}$ and who received appropriate treatment. Electronic records were parsed for clinical factors including receipt of PPIs, other acid suppression, non-CDI antibiotics, and comorbidities. The primary exposure was in-hospital PPIs given concurrently with $C$. difficile treatment. Recurrence was defined as a second positive stool test 15 to 90 days after the initial positive test. $C$. difficile recurrence rates in the PPI exposed and unexposed groups were compared with the log-rank test. Multivariable Cox proportional hazards modeling was performed to control for demographics, comorbidities, and other clinical factors.
\end{abstract}

Results-We identified 894 inpatients with incident CDI. The cumulative incidence of CDI recurrence in the cohort was $23 \%$. Receipt of PPIs concurrent with CDI treatment was not associated with $C$. difficile recurrence (HR 0.82; 95\% CI 0.58-1.16). Black race (HR 1.66, 95\% CI 1.05-2.63), increased age (HR 1.02, 95\% CI 1.01-1.03), and increased comorbidities (HR 1.09, 95\% CI 1.04-1.14) were associated with CDI recurrence. In light of a higher 90-day mortality seen among those who received PPIs ( $\log$-rank $\mathrm{p}=0.02$ ), we also analyzed the subset of patients who survived to 90 days of follow-up. Again, there was no association between PPIs and CDI

Correspondence: Daniel E. Freedberg, MD, 630 West $168^{\text {th }}$ Street, PH Bldg Floor 20, New York, NY 10032, USA.

def2004@columbia.edu.

\section{CONFLICT OF INTEREST}

Guarantor of the article: Daniel E. Freedberg, MD

Specific author contributions: Conceived and designed the study, acquired data, analyzed and interpreted the data, drafted the manuscript, had full access to all of the data in the study and takes responsibility for the integrity of the data and the accuracy of the data analysis: DEF; conceived and designed the study, supervised the analysis, interpreted the data, and critically revised the manuscript: JAA; acquired data and critically revised the manuscript: HS; critically revised the manuscript: CF.

Financial support: Dr. Freedberg was supported in part by a training grant from the National Institute of Diabetes and Digestive and Kidney Diseases (T32 DK083256-0). Dr. Salmasian and Dr. Friedman were supported in part by National Library of Medicine grants R01 LM010016, R01 LM010016-0S1, R01 LM010016-0S2, R01 LM008635, and T15 LM007079. Dr. Abrams was supported in part by a Career Development Award from the National Cancer Institute (K07 CA 132892).

Potential competing interests: Carol Friedman is a consultant for a company that licenses the MedLEE natural language processing system. 
recurrence (HR 0.87; 95\% CI 0.60-1.28). Finally, there was no association between recurrent CDI and increased duration or dose of PPIs.

Conclusions-Among hospitalized adults with $C$. difficile, receipt of PPIs concurrent with $C$. difficile treatment was not associated with CDI recurrence. Black race, increased age, and increased comorbidities significantly predicted recurrence. Future studies should test interventions to prevent $\mathrm{CDI}$ recurrence among high risk inpatients.

\section{INTRODUCTION}

Proton pump inhibitors (PPIs) are a risk factor for incident Clostridium difficile infection (CDI) ${ }^{1-3}$ PPIs are among the most common drugs in the world; in America, esomeprazole was the third most prescribed drug by sales in $2011 .{ }^{4}$ They are highly effective in treating gastric acid-related disorders ${ }^{1}$ but are often prescribed without a documented indication. 5,6 Other established risk factors for CDI include older age, antibiotics, hospitalization, and gastrointestinal tract abnormalities; ${ }^{7,8}$ PPIs appear to act synergistically with other risk factors to increase risk of incident $C$. difficile among both inpatients and outpatients. ${ }^{9,10}$

Up to $30 \%$ of patients with CDI recur after completing treatment ${ }^{11}$ and limited data suggests that PPIs may be a risk factor for recurrent as well as incident CDI. A study combining inand outpatients at 8 Veterans Affairs medical centers in New England suggested that PPIs were associated with a moderately increased risk of recurrent CDI. ${ }^{12}$ Two smaller studies reached similar conclusions although with heterogeneity in their estimates of risk. ${ }^{13,14}$

The factors that influence $C$. difficile recurrence differ between in- and outpatients. Inpatients with incident CDI are older, have more comorbidities, and are more often exposed to antibiotics compared to outpatients. ${ }^{15}$ Inpatients with CDI are more likely to have been exposed to PPIs compared to outpatients; when PPIs are given, there are differences between in- and outpatients in indications for use, duration, dosage, and method of administration. ${ }^{16}$ Furthermore, inpatients have more severe $C$. difficile and are more likely to be exposed to hypervirulent subtypes such as the North American Pulsed Field type 1 strain. ${ }^{17,18}$

For these reasons, factors that influence Clostridium difficile recurrence may have distinct strengths of association in the inpatient setting as opposed to the outpatient setting. Yet studies to date have not focused on PPIs as a risk factor for recurrence exclusively among inpatients with CDI. We therefore sought to study the relationship between in-hospital use of PPIs and recurrent CDI in a retrospective cohort analysis of inpatients with Clostridium difficile infection.

\section{METHODS}

\section{Study population}

We electronically reviewed the medical records from all adult inpatients at our institution testing positive for C. difficile from September 1, 2009 to June 30, 2012. (September 1, 2009 was the day on which our institution switched from a $C$. difficile immunoassay to the stool polymerase chain reaction (PCR) test for $C$. difficile toxin B.) From this group, we identified all patients with incident CDI, defined as a positive stool PCR test while hospitalized from December 1, 2009 to June 30, 2012 without a prior positive test within 90 days who were treated for $C$. difficile. To minimize loss to follow-up, we excluded from the analysis patients who did not meet study endpoints or did not have proven clinical follow-up in our medical system as evidenced by a clinical encounter after the 90-day study period (Figure 
1). The Institutional Review Board of Columbia University Medical Center approved this study.

\section{Measures}

Using automated electronic queries, we extracted information regarding age, sex, selfreported race/ethnicity, length of stay, and hospitalization in an intensive care unit (ICU) during the index admission. Death was extracted from the electronic medical record (EMR) which is cross-indexed with the National Social Security Death Index. We additionally extracted information regarding medications received during the $C$. difficile treatment period including PPIs, acid suppression with a histamine-2 receptor antagonist (H2RA), type of $C$. difficile treatment, non-CDI antibiotics, and immunosuppressants. Discharge summaries were manually reviewed to extract information regarding discharge PPIs or other acid suppression, non-CDI antibiotics, and immunosuppressants. However, because discharge summaries were frequently missing or incomplete $(n=181)$, we ultimately did not include these variables in the analyses. Patients receiving solely H2RAs for gastric acid suppression were classified as having received H2RAs; those receiving H2RAs concurrent with PPIs were classified as having received PPIs. Type of $C$. difficile treatment was categorized as metronidazole alone, vancomycin alone, or both medications; none of the patients in the study received fidaxomicin. Steroids were considered to be immunosuppressive only if prescribed at a dose of at least $5 \mathrm{mg}$ prednisone daily or equivalent.

Our EMR uses standard International Classification of Diseases (ICD) coding to describe patient comorbidities. We found that ICD coding of patient comorbidities was inaccurate compared to manual review, a phenomenon that has been previously described. ${ }^{19,20}$ To avoid the limitations of ICD codes we used a natural language processing (NLP) system called Medical Language Extraction and Encoding (MedLEE) to electronically process admission notes and extract patient comorbidities. ${ }^{21}$ MedLEE is a rule-based program that transforms natural language clinical text into computable data; ${ }^{22-24}$ here MedLEE captured comorbidity variables with $88 \%$ accuracy compared to a subset manual chart review. ${ }^{25}$ Comorbidities were described in a modified Charlson comorbidity index ${ }^{26}$ omitting tumors with/without metastases and mild liver disease because these distinctions could not be clearly made from existing records.

\section{Primary exposure and outcome}

The primary exposure was defined a priori as in-hospital PPI therapy at any dose and at least daily in frequency for a minimum of two days during the $C$. difficile treatment period. The primary outcome was defined as a second positive PCR for the $C$. difficile toxin B gene within 15 to 90 days after the initial positive test. The stool PCR test for the toxin B gene is $93 \%$ sensitive and $97 \%$ specific. $^{27,28}$ To select the recurrence window of 15 to 90 days, we referred to studies suggesting that 1 ) most patients respond to $C$. difficile treatment within 3 to 4 days and over $90 \%$ are cured after 14 days of standard treatment ${ }^{29,30}$ and 2) CDI recurrence risk peaks 7 to 30 days after cessation of antibiotics but persists for up to 90 days. ${ }^{31,32}$ Patients were censored at death or 90 days after their initial positive test.

In order to determine the duration of exposure to PPIs, we manually reviewed patients' electronic medical records to identify the clinical encounters preceding the date of recurrence (among those who had recurrent CDI) or the date of censoring (among those who did not have recurrent CDI). We then recategorized PPI duration as follows: none, partial duration (PPIs given either concurrent with $C$. difficile treatment or during the follow-up period but not during both times), and full duration (PPIs given concurrent with $C$. difficile treatment and during follow-up). In order to determine the relationship between strength of acid suppression and recurrent CDI, we defined four categories of acid suppression given 
during $C$. difficile treatment: no PPIs, solely H2RAs, low dose PPIs (equivalent to esomeprazole 20mg daily or less), and high dose PPIs (equivalent to more than esomeprazole $20 \mathrm{mg}$ daily).

\section{Statistical analysis}

Categorical variables were analyzed using chi-squared tests (for data satisfying the central limit theorum) or Fisher's exact tests and continuous variables were analyzed using t-tests (for normally distributed data) or Wilcoxon rank sum tests. $C$. difficile recurrence rates in the PPI exposed and unexposed groups were compared with the log-rank test. Multivariable Cox proportional hazards modeling was performed to assess the association between PPIs and CDI recurrence, controlling for potential confounders. We included the following variables in the model: age, sex, race/ethnicity, comorbidities (modified Charlson score), type of $C$. difficile treatment, receipt of antibiotics, receipt of immunosuppressants, ICU stay, and hospital length of stay. In post hoc analyses we examined the subset of patients who survived to complete 90 days of follow-up and assessed for associations between PPI duration and recurrent CDI and between PPI dose and recurrent CDI. All data were analyzed using SAS 9.3 (SAS Institute, Cary, NC) at the alpha 0.05 level of significance.

\section{RESULTS}

\section{Patient characteristics}

We identified 894 hospitalized patients with a newly positive stool PCR test for $C$. difficile toxin B between December 1, 2009 and June 30, 2012 and documented follow-up in our medical system. Overall, $62 \%$ of patients received PPIs concurrently with CDI treatment. Only 32 patients (4\%) received acid suppression with H2RAs alone. Over $98 \%$ of patients receiving PPIs were given esomeprazole. Patient received similar rates of PPIs whether they were among those analyzed or among the 323 patients who did not have follow-up in our medical system $(\mathrm{p}=0.50)$. Among those with recurrent CDI, 93 of $167(56 \%)$ were receiving a PPI at the time of the diagnosis of CDI recurrence; among those who did not have recurrent CDI, 406 of $727(56 \%)$ were receiving a PPI at the time of censoring. Among the 167 patients with recurrent CDI, 33 patients (20\%) recurred during the index admission with a median time to recurrence of 33 days (range 15 to 67 days). An additional 66 patients (40\%) were discharged but recurred during a subsequent admission while 68 patients $(41 \%)$ recurred as outpatients.

\section{Baseline and hospitalization-related risk factors}

At the time of hospital admission, patient demographic and clinical characteristics were similar between those who did and did not receive PPIs concurrent with CDI treatment (Table 1). However, during hospitalization patients who received PPIs were more likely to receive antibiotics and immunosuppressants compared to patients who did not receive PPIs and were more likely to be treated for $C$. difficile with both metronidazole and vancomycin, a potential indication of severe disease. Additionally, patients who were given PPIs during C. difficile treatment had significantly longer hospitalizations, were more likely to be hospitalized in an ICU, and more likely to die within 90 days compared to patients who were not given PPIs (Table 2).

\section{Multivariable analysis}

The primary outcome of recurrent CDI occurred in 167 patients with a cumulative incidence of 22\% among those who received PPIs concurrent with $C$. difficile treatment compared to $24 \%$ among those who did not receive PPIs (log-rank $\mathrm{p}=0.56)($ Figure 2$)$. In multivariable analysis, the adjusted hazard ratio for PPIs was 0.82 (95\% CI 0.58-1.16) (Table 3). When 
acid suppression with H2RAs was added to the model to assess for a dose-related effect (subjects categorized as no acid suppression, H2RAs only, or PPIs), there was no change in the relationship between PPIs and CDI recurrence and no association between receipt of H2RAs and CDI recurrence. Black race was identified as an independent predictor of CDI recurrence (HR 1.66, 95\% CI 1.05-2.63). Older age and increased comorbidities at the time of admission were also significantly associated with CDI recurrence.

\section{Sensitivity analysis}

The cumulative 90-day mortality within the population was significantly higher among patients who received PPIs compared to those who did not $(\log$-rank $\mathrm{p}=0.02)$ although PPIs were not associated with mortality in a multivariable analysis (HR $0.95,95 \%$ CI $0.71-1.27$ ). To assess the possibility of death as a competing risk, we examined the patients who survived through 90 days of follow-up after the initial positive test. Among this subset of 635 patients there was no association between PPIs and CDI recurrence (HR 0.87, 95\% CI $0.60-1.28$ ). Because antibiotic-associated diarrhea could result in increased testing for $C$. difficile, we performed an analysis stratified by receipt of antibiotics. Again there was no association between receipt of PPIs and CDI recurrence among 653 patients who received antibiotics (HR $0.79,95 \%$ CI $0.53-1.17$ ) or among 241 patients who did not (HR 1.00, 95\% CI 0.48-2.09).

There was no association between increased duration of PPIs and recurrent CDI. Compared to patients who did not receive PPIs, there was no association between partial duration PPIs and CDI recurrence (HR 0.94; 95\% CI 0.61-1.44) or between full duration PPIs and CDI recurrence (HR 0.91; 95\% CI 0.62-1.34). Also, there was no association between increased strength of acid suppression and recurrent CDI. Overall, 32 patients $(4 \%)$ received H2RAs only, 415 patients (46\%) received low dose PPIs and 136 patients (15\%) received high dose PPIs. When strength of acid suppression was included in the multivariable model, there was no association between CDI recurrence and solely H2RAs (HR 1.86, 95\% CI 0.94-3.69), low dose PPIs (HR 0.86, 95\% CI 0.59-1.26), or high dose PPIs (HR 0.98, 95\% CI 0.60$1.60)$ and no evidence of a trend between increased acid suppression and CDI recurrence ( $\mathrm{p}$ $=0.57$ ).

\section{DISCUSSION}

Among 894 hospitalized patients with $C$. difficile at our institution, we found no association between receipt of PPIs during $C$. difficile treatment and CDI recurrence within 15 to 90 days after the initial positive test. This result remained null in a post hoc subset analysis of patients who survived to complete 90 days of follow-up and in analyses based on duration and dose of PPI exposure. Interestingly, black race was identified as an independent predictor of recurrence. Increased age and increased comorbidities were also associated with CDI recurrence.

Multiple observational studies have suggested that PPIs are a risk factor for incident CDI yet doubt remains as to whether PPIs are truly causal in $C$. difficile or whether the observed relationship is due to unmeasured confounding. ${ }^{33,34} \mathrm{By}$ focusing exclusively on inpatients with CDI, the current study minimizes clinical heterogeneity between patients who did and did not receive PPIs. In addition to PPIs, other risk factors for incident CDI include older age, antibiotics, hospitalization, and gastrointestinal tract abnormalities. ${ }^{8}$ Previously identified risk factors for recurrent CDI include older age and antibiotics as well as use of acid-suppressing medicines. ${ }^{35-37}$ Our data support older age as an independent risk factor for recurrent CDI. Three prior studies have found that PPIs were associated with a moderately increased likelihood of CDI recurrence. ${ }^{12-14}$ Ascertainment of comorbidities, definitions of PPI exposure, and the populations under consideration all differ between our 
study and prior studies. Although methodological differences make it challenging to compare populations, our patients appear to have had more severe CDI as evidenced by the large portion $(41 \%)$ who were treated with both metronidazole and vancomycin compared to under $10 \%$ in the other studies. It is possible that the pathogenesis of CDI recurrence varies between patients with severe CDI and relatively healthier patients with $C$. difficile.

This is the first study to find that black race may be an independent risk factor for recurrent CDI. Previous studies have suggested that black race may be protective of incident $\mathrm{CDI}^{38-40}$ whereas prior studies of recurrent CDI have not assessed race. ${ }^{12-14}$ The observed association between black race and CDI recurrence in the present study may be mediated by factors that influence the microbiome such as diet, biological differences such as a difference in mean serum albumin level, ${ }^{41}$ or socioeconomic factors such as insurance status, access to medicines, or nursing home residency status. Future studies are warranted to identify the specific factors responsible for the observed association between black race and $C$. difficile recurrence.

Although our study does not support an association between PPIs and recurrent $C$. difficile, there is robust observational data supporting an association between PPIs and incident $C$. difficile. The mechanism by which PPIs might cause incident CDI is uncertain. ${ }^{1}$ C. difficile spores are acid resistant and acid suppression has little impact on their survival. ${ }^{42,43}$ However, gastric acid suppression with PPIs may alter the colonic microbiome to decrease colonization resistance or other normal barriers to $C$. difficile proliferation. The microbiome is vital in maintaining normal immunity ${ }^{44}$ and in animals PPIs alter the colonic microbiome in a manner similar to antibiotics. ${ }^{45-47}$ It is biologically plausible that PPIs might contribute to incident CDI by perturbing the microbiome yet have little effect on CDI recurrence because the microbiome of patients at risk for recurrence is already altered.

This study has multiple strengths. This is the first large study to focus exclusively on inpatients with CDI who receive PPIs. Clostridium difficile infection is often a nosocomial disease and the factors driving in- versus outpatient CDI are different; this study directly addresses the question of whether inpatients with CDI can be given PPIs during $C$. difficile treatment without increasing risk for CDI recurrence. Because all patients were hospitalized, we were able to ascertain primary exposure and outcome with high confidence. We extracted comorbidities from the medical record using natural language processing, which is more accurate than ICD coding. ${ }^{22-24} \mathrm{We}$ also evaluated a time period during which $C$. difficile diagnosis was based on stool PCR testing, which has improved test parameters compared to earlier-generation immunoassays. ${ }^{28}$ Our study is relatively large, incorporates time to recurrence in the primary analysis, and adequately assesses other possible explanations for recurrence of CDI including patient risk factors, antibiotics, and other acid suppression.

There are some limitations to our study. Diarrhea is a common side effect of PPIs and could lead to increased testing for $C$. difficile. However, increased testing among patients who received PPIs would have biased findings away from the null, towards a positive association between PPIs and recurrent CDI. While our study was relatively large, it was not powered to detect very small differences in CDI recurrence rates among patients exposed to PPIs compared to patients who were not exposed. Our patients had a high degree of comorbidity and our results may not be generalizable to healthier inpatient populations. Despite exclusion of patients who lacked adequate clinical follow-up, we do not have a closed hospital system and patients may have had recurrence diagnosed elsewhere. However, our methods were designed to minimize such losses, there was no evidence of differential loss to follow-up by receipt of PPIs, and our overall recurrence rate was similar to prior studies. ${ }^{12,48}$ Patients in our study who received PPIs received almost exclusively 
esomeprazole. However, there is no evidence that type of PPI modifies the relationship between PPIs and CDI; we believe that our findings regarding esomeprazole and recurrent CDI are indicative of a class effect.

In sum, among hospitalized patients with newly diagnosed Clostridium difficile, administration of PPIs concurrently with $C$. difficile treatment was not a risk factor for CDI recurrence. Black race, increased age, and additional comorbidities were predictive of recurrence. Our primary results are best applied to populations that resemble the patients in our study - patients in tertiary care settings with a high degree of illness and multiple comorbidities. While hospitalized patients frequently receive PPIs without indication, inhospital administration of PPIs does not increase the risk of CDI recurrence. Future studies should test interventions to prevent CDI recurrence among high risk inpatients.

\section{Acknowledgments}

Dario Mercedes provided programming assistance.

\section{References}

1. Yang YX, Metz DC. Safety of proton pump inhibitor exposure. Gastroenterology. 2010; 139:11151127. [PubMed: 20727892]

2. Janarthanan S, Ditah I, Adler DG, et al. Clostridium difficile-associated diarrhea and proton pump inhibitor therapy: a meta-analysis. Am J Gastroenterol. 2012; 107:1001-1010. [PubMed: 22710578]

3. Kwok CS, Arthur AK, Anibueze CI, et al. Risk of Clostridium difficile infection with acid suppressing drugs and antibiotics: meta-analysis. Am J Gastroenterol. 2012; 107:1011-1019. [PubMed: 22525304]

4. IMS Health NSP. [Accessed January 24, 2013] Feb 23. 2012 http://www.imshealth.com/ims/Global/ Content/Corporate/Press\%20Room/Top-Line\%20Market\%20Data\%20\&\%20Trends/2011\%20Topline\%20Market\%20Data/Top_Products_by_Sales.pdf

5. Bashford JN, Norwood J, Chapman SR. Why are patients prescribed proton pump inhibitors? Retrospective analysis of link between morbidity and prescribing in the General Practice Research Database. BMJ. 1998; 317:452-456. [PubMed: 9703528]

6. Jacobson BC, Ferris TG, Shea TL, et al. Who is using chronic acid suppression therapy and why? Am J Gastroenterol. 2003; 98:51-58. [PubMed: 12526936]

7. Bartlett JG. Clinical practice. Antibiotic-associated diarrhea. N Engl J Med. 2002; 346:334-349. [PubMed: 11821511]

8. Surawicz CM, Brandt LJ, Binion DG, et al. Guidelines for Diagnosis, Treatment, and Prevention of Clostridium difficile Infections. Am J Gastroenterol. 2013; 108:478-498. [PubMed: 23439232]

9. Howell MD, Novack V, Grgurich P, et al. Iatrogenic gastric acid suppression and the risk of nosocomial Clostridium difficile infection. Arch Intern Med. 2010; 170:784-790. [PubMed: 20458086]

10. Dial S, Delaney JA, Barkun AN, et al. Use of gastric acid-suppressive agents and the risk of community-acquired Clostridium difficile-associated disease. JAMA. 2005; 294:2989-2995. [PubMed: 16414946]

11. Maroo S, Lamont JT. Recurrent clostridium difficile. Gastroenterology. 2006; 130:1311-1316. [PubMed: 16618421]

12. Linsky A, Gupta K, Lawler EV, et al. Proton pump inhibitors and risk for recurrent Clostridium difficile infection. Arch Intern Med. 2010; 170:772-778. [PubMed: 20458084]

13. Kim JW, Lee KL, Jeong JB, et al. Proton pump inhibitors as a risk factor for recurrence of Clostridium-difficile-associated diarrhea. World J Gastroenterol. 2010; 16:3573-3577. [PubMed: 20653067]

14. Cadle RM, Mansouri MD, Logan N, et al. Association of proton-pump inhibitors with outcomes in Clostridium difficile colitis. Am J Health Syst Pharm. 2007; 64:2359-2363. [PubMed: 17989446] 
15. Khanna S, Pardi DS, Aronson SL, et al. The epidemiology of community-acquired Clostridium difficile infection: a population-based study. Am J Gastroenterol. 2012; 107:89-95. [PubMed: 22108454]

16. Heidelbaugh JJ, Goldberg KL, Inadomi JM. Overutilization of proton pump inhibitors: a review of cost-effectiveness and risk [corrected]. Am J Gastroenterol. 2009; 104(Suppl 2):S27-S32. [PubMed: 19262544]

17. Kelly CP, LaMont JT. Clostridium difficile--more difficult than ever. N Engl J Med. 2008; 359:1932-1940. [PubMed: 18971494]

18. Gravel D, Miller M, Simor A, et al. Health care-associated Clostridium difficile infection in adults admitted to acute care hospitals in Canada: a Canadian Nosocomial Infection Surveillance Program Study. Clin Infect Dis. 2009; 48:568-576. [PubMed: 19191641]

19. Wright A, Maloney FL, Feblowitz JC. Clinician attitudes toward and use of electronic problem lists: a thematic analysis. BMC Med Inform Decis Mak. 2011; 11:36. [PubMed: 21612639]

20. Szeto HC, Coleman RK, Gholami P, et al. Accuracy of computerized outpatient diagnoses in a Veterans Affairs general medicine clinic. Am J Manag Care. 2002; 8:37-43. [PubMed: 11814171]

21. Chuang JH, Friedman C, Hripcsak G. A comparison of the Charlson comorbidities derived from medical language processing and administrative data. Proc AMIA Symp. 2002:160-164. [PubMed: 12463807]

22. Hripcsak G, Friedman C, Alderson PO, et al. Unlocking clinical data from narrative reports: a study of natural language processing. Ann Intern Med. 1995; 122:681-688. [PubMed: 7702231]

23. Murff HJ, FitzHenry F, Matheny ME, et al. Automated identification of postoperative complications within an electronic medical record using natural language processing. JAMA. 2011; 306:848-855. [PubMed: 21862746]

24. Salmasian H, Freedberg DE, Abrams JA, et al. An automated tool for detecting medication overuse based on the electronic health records. Pharmacoepidemiol Drug Saf. 2012

25. Salmasian H, Freedberg DE, Friedman C. J Am Med Inform Assoc. under review.

26. Charlson ME, Pompei P, Ales KL, et al. A new method of classifying prognostic comorbidity in longitudinal studies: development and validation. J Chronic Dis. 1987; 40:373-383. [PubMed: 3558716]

27. Huang H, Weintraub A, Fang H, et al. Comparison of a commercial multiplex real-time PCR to the cell cytotoxicity neutralization assay for diagnosis of clostridium difficile infections. J Clin Microbiol. 2009; 47:3729-3731. [PubMed: 19741082]

28. Peterson LR, Manson RU, Paule SM, et al. Detection of toxigenic Clostridium difficile in stool samples by real-time polymerase chain reaction for the diagnosis of C. difficile-associated diarrhea. Clin Infect Dis. 2007; 45:1152-1160. [PubMed: 17918076]

29. Nair S, Yadav D, Corpuz M, et al. Clostridium difficile colitis: factors influencing treatment failure and relapse--a prospective evaluation. Am J Gastroenterol. 1998; 93:1873-1876. [PubMed: 9772047]

30. Wenisch C, Parschalk B, Hasenhundl M, et al. Comparison of vancomycin, teicoplanin, metronidazole, and fusidic acid for the treatment of Clostridium difficile-associated diarrhea. Clin Infect Dis. 1996; 22:813-818. [PubMed: 8722937]

31. Hensgens MP, Goorhuis A, Dekkers OM, et al. Time interval of increased risk for Clostridium difficile infection after exposure to antibiotics. J Antimicrob Chemother. 2012; 67:742-748. [PubMed: 22146873]

32. Dial S, Kezouh A, Dascal A, et al. Patterns of antibiotic use and risk of hospital admission because of Clostridium difficile infection. CMAJ. 2008; 179:767-772. [PubMed: 18838451]

33. Leontiadis GI, Miller MA, Howden CW. How much do PPIs contribute to C. difficile infections? Am J Gastroenterol. 2012; 107:1020-1021. [PubMed: 22764024]

34. Johnson DA, Oldfield ECt. Reported Side Effects and Complications of Long-term Proton Pump Inhibitor Use: Dissecting the Evidence. Clin Gastroenterol Hepatol. 2013; 11:458-464. [PubMed: 23247326]

35. Garey KW, Sethi S, Yadav Y, et al. Meta-analysis to assess risk factors for recurrent Clostridium difficile infection. J Hosp Infect. 2008; 70:298-304. [PubMed: 18951661] 
36. Pepin J, Saheb N, Coulombe MA, et al. Emergence of fluoroquinolones as the predominant risk factor for Clostridium difficile-associated diarrhea: a cohort study during an epidemic in Quebec. Clin Infect Dis. 2005; 41:1254-1260. [PubMed: 16206099]

37. Tal S, Gurevich A, Guller V, et al. Risk factors for recurrence of Clostridium difficile-associated diarrhea in the elderly. Scand J Infect Dis. 2002; 34:594-597. [PubMed: 12238576]

38. Tsiouris A, Neale JA, Reickert CA, et al. Clostridium difficile of the ileum following total abdominal colectomy, with or without proctectomy: who is at risk? Dis Colon Rectum. 2012; 55:424-428. [PubMed: 22426266]

39. Murphy CR, Avery TR, Dubberke ER, et al. Frequent hospital readmissions for Clostridium difficile infection and the impact on estimates of hospital-associated C. difficile burden. Infect Control Hosp Epidemiol. 2012; 33:20-28. [PubMed: 22173518]

40. Doernberg SB, Winston LG, Deck DH, et al. Does doxycycline protect against development of Clostridium difficile infection? Clin Infect Dis. 2012; 55:615-620. [PubMed: 22563022]

41. Shakov R, Salazar RS, Kagunye SK, et al. Diabetes mellitus as a risk factor for recurrence of Clostridium difficile infection in the acute care hospital setting. Am J Infect Control. 2011; 39:194-198. [PubMed: 21349600]

42. Rao A, Jump RL, Pultz NJ, et al. In vitro killing of nosocomial pathogens by acid and acidified nitrite. Antimicrob Agents Chemother. 2006; 50:3901-3904. [PubMed: 17065628]

43. Wilson KH, Sheagren JN, Freter R. Population dynamics of ingested Clostridium difficile in the gastrointestinal tract of the Syrian hamster. J Infect Dis. 1985; 151:355-361. [PubMed: 3968453]

44. Willing BP, Russell SL, Finlay BB. Shifting the balance: antibiotic effects on host-microbiota mutualism. Nat Rev Microbiol. 2011; 9:233-243. [PubMed: 21358670]

45. Jakobsson HE, Jernberg C, Andersson AF, et al. Short-term antibiotic treatment has differing longterm impacts on the human throat and gut microbiome. PLoS One. 2010; 5:e9836. [PubMed: 20352091]

46. Garcia-Mazcorro JF, Suchodolski JS, Jones KR, et al. Effect of the proton pump inhibitor omeprazole on the gastrointestinal bacterial microbiota of healthy dogs. FEMS Microbiol Ecol. 2012; 80:624-636. [PubMed: 22324305]

47. Manges AR, Labbe A, Loo VG, et al. Comparative metagenomic study of alterations to the intestinal microbiota and risk of nosocomial Clostridum difficile-associated disease. J Infect Dis. 2010; 202:1877-1884. [PubMed: 21050115]

48. Fekety R, McFarland LV, Surawicz CM, et al. Recurrent Clostridium difficile diarrhea: characteristics of and risk factors for patients enrolled in a prospective, randomized, doubleblinded trial. Clin Infect Dis. 1997; 24:324-333. [PubMed: 9114180] 


\section{STUDY HIGHLIGHTS}

\section{What is current knowledge}

- C. difficile infection is a common nosocomial disease with high associated morbidity and mortality.

- C. difficile recurrence occurs in up to one third of patients and is predicted by older age and the presence of comorbidities.

- PPIs are a risk factor for incident CDI; limited data suggests that PPIs may also be a risk factor for recurrent CDI.

\section{What is new here}

- In-hospital administration of PPIs did not associate with recurrence of CDI among inpatients.

- Black race, older age, and increased comorbidities are significant predictors of CDI recurrence. 


\section{Adult inpatients testing positive for \\ C. difficile toxin B $(n=1260)$}

Prior positive test $(n=25)$

Lack of follow-up $(n=323)$

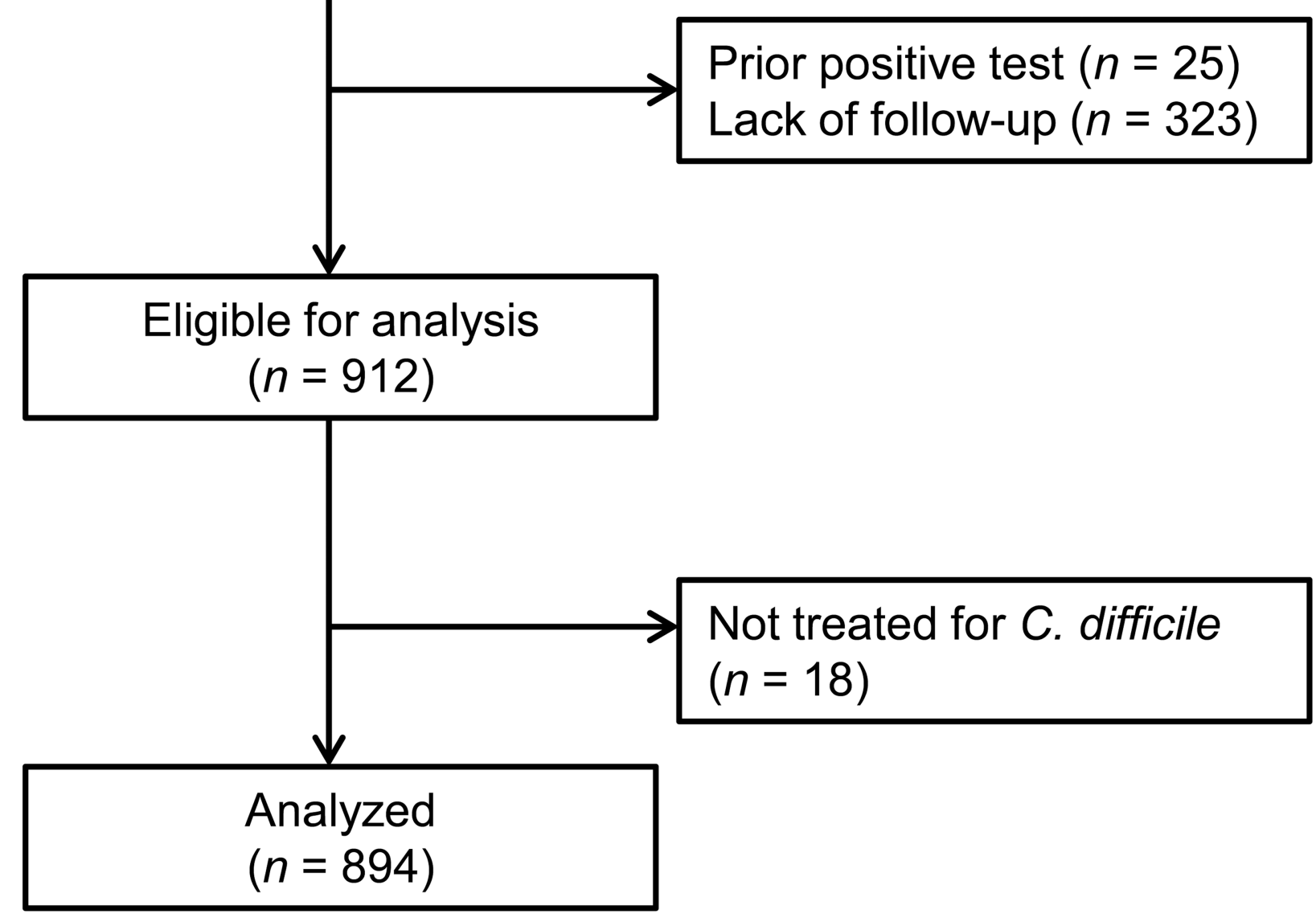

Figure 1.

Flow diagram of patients evaluated and analyzed for association between proton pump inhibitors and recurrent Clostridium difficile infection. 


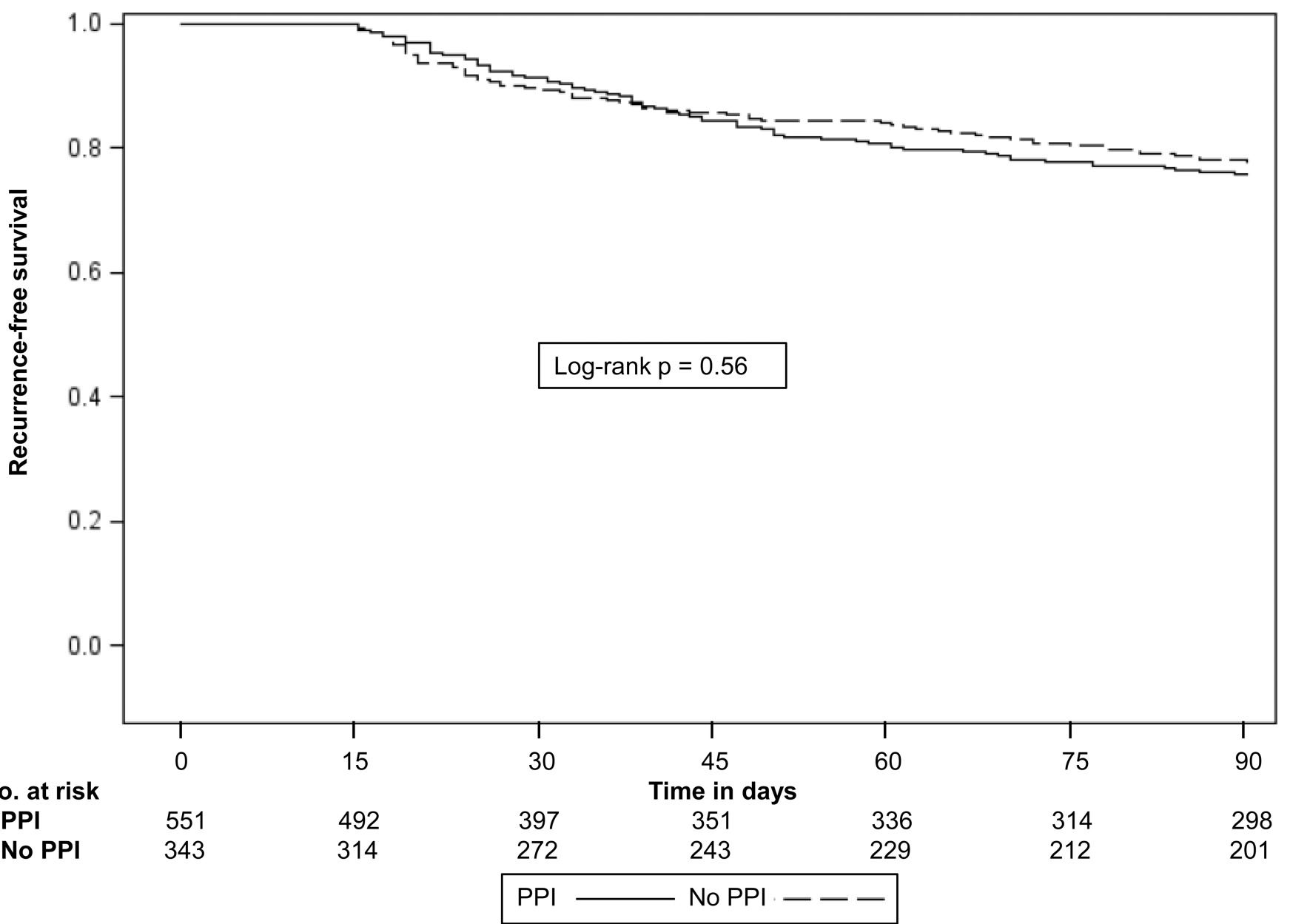

Figure 2.

Kaplan-Meier plot of Clostridium difficile recurrence among patients who did and did not receive proton pump inhibitors during treatment for initial $C$. difficile infection. 
Table 1

Baseline demographic and clinical characteristics stratified by proton pump inhibitors given concurrently with treatment for Clostridium difficile

\begin{tabular}{|c|c|c|c|c|}
\hline Characteristics & $\begin{array}{l}\text { All } \\
n=894\end{array}$ & $\begin{array}{l}\text { PPIs } \\
\mathrm{n}=551\end{array}$ & $\begin{array}{l}\text { No PPIs } \\
n=343\end{array}$ & p-value \\
\hline Age in years (mean, $\pm \mathrm{SD}$ ) & $64 \pm 19$ & $64 \pm 18$ & $65 \pm 20$ & 0.43 \\
\hline Male sex & $431(48 \%)$ & $266(48 \%)$ & $165(48 \%)$ & 1.00 \\
\hline Race/ethnicity & & & & 0.66 \\
\hline White & $278(31 \%)$ & $176(32 \%)$ & $102(30 \%)$ & \\
\hline Black & $105(12 \%)$ & $60(11 \%)$ & $45(13 \%)$ & \\
\hline Hispanic & $306(34 \%)$ & $192(35 \%)$ & $114(33 \%)$ & \\
\hline Unknown/other & $205(23 \%)$ & $123(22 \%)$ & $82(24 \%)$ & \\
\hline Charlson index (median, IQR) ${ }^{a}$ & $2(0-5)$ & $2(0-5)$ & $2(0-5)$ & 0.82 \\
\hline \multicolumn{5}{|l|}{ Comorbidities } \\
\hline MI & $189(21 \%)$ & $121(22 \%)$ & $68(20 \%)$ & 0.45 \\
\hline $\mathrm{CHF}$ & $222(25 \%)$ & $144(26 \%)$ & $78(23 \%)$ & 0.25 \\
\hline PVD & $77(9 \%)$ & $49(9 \%)$ & $28(8 \%)$ & 0.71 \\
\hline Stroke & $219(25 \%)$ & $130(24 \%)$ & $89(26 \%)$ & 0.43 \\
\hline Dementia & $116(13 \%)$ & $51(9 \%)$ & $65(19 \%)$ & $<0.01$ \\
\hline COPD & $47(5 \%)$ & $33(6 \%)$ & $14(4 \%)$ & 0.21 \\
\hline Connective tissue disease & $55(6 \%)$ & $37(7 \%)$ & $18(5 \%)$ & 0.38 \\
\hline PUD & $72(8 \%)$ & $54(10 \%)$ & $18(5 \%)$ & 0.02 \\
\hline Cirrhosis & $93(10 \%)$ & $63(11 \%)$ & $30(9 \%)$ & 0.20 \\
\hline Diabetes & $311(35 \%)$ & $207(37 \%)$ & $109(32 \%)$ & 0.14 \\
\hline Hemiplegia & $59(7 \%)$ & $33(6 \%)$ & $26(8 \%)$ & 0.35 \\
\hline CKD & $245(27 \%)$ & $152(28 \%)$ & $93(27 \%)$ & 0.88 \\
\hline Leukemia & $28(3 \%)$ & $13(2 \%)$ & $15(4 \%)$ & 0.09 \\
\hline Lymphoma & $42(5 \%)$ & $26(5 \%)$ & $16(5 \%)$ & 0.97 \\
\hline AIDS & $63(7 \%)$ & $34(6 \%)$ & $29(8 \%)$ & 0.19 \\
\hline
\end{tabular}

Abbreviations: SD, standard deviation; IQR, interquartile range; MI, myocardial infarction; CHF, congestive heart failure; PVD, peripheral vascular disease; COPD, congestive-obstructive pulmonary disease; PUD, peptic ulcer disease; CKD, chronic kidney disease; AIDS, acquired immunodeficiency syndrome.

${ }^{a}$ Modified Charlson index as described in the text. 
Table 2

Hospitalization characteristics stratified by proton pump inhibitors given concurrently with treatment for Clostridium difficile

\begin{tabular}{|lllll|}
\hline Risk factors & $\begin{array}{l}\text { All } \\
\mathbf{n = 8 9 4}\end{array}$ & $\begin{array}{l}\text { PPIs } \\
\mathbf{n = 5 5 1}\end{array}$ & $\begin{array}{l}\text { No PPIs } \\
\mathbf{n = 3 4 3}\end{array}$ & p-value \\
\hline Medications & & & & \\
Antibiotics & $653(73 \%)$ & $476(86 \%)$ & $177(52 \%)$ & $<0.01$ \\
Immunosuppressants & $311(35 \%)$ & $260(47 \%)$ & $51(15 \%)$ & $<0.01$ \\
C. difficile treatment & & & & 0.04 \\
Metronidazole & $458(51 \%)$ & $266(48 \%)$ & $192(56 \%)$ & \\
Vancomycin & $70(8 \%)$ & $41(7 \%)$ & $29(8 \%)$ & \\
Both & $366(41 \%)$ & $244(45 \%)$ & $122(36 \%)$ & \\
Morbidity & & & & \\
ICU stay & $381(43 \%)$ & $290(53 \%)$ & $91(27 \%)$ & $<0.01$ \\
Death within 90 days & $259(29 \%)$ & $174(32 \%)$ & $85(25 \%)$ & 0.03 \\
Hospital LOS in days (median, IQR) & $13(7-25)$ & $17(9-31)$ & $8(5-16)$ & $<0.01$ \\
\hline
\end{tabular}

Abbreviations: ICU, intensive care unit; LOS, length of stay; IQR, interquartile range. 
Table 3

Multivariable model of risk factors for recurrence of Clostridium difficile infection within 15-90 days of initial positive test

\begin{tabular}{|c|c|c|}
\hline Risk factors & HR & $95 \% \mathrm{CI}$ \\
\hline \multicolumn{3}{|l|}{ PPIs } \\
\hline No $(n=343)$ & Ref & --- \\
\hline Yes $(n=551)$ & 0.82 & $0.58-1.16$ \\
\hline \multicolumn{3}{|l|}{ Patient characteristics } \\
\hline Age, per additional year & 1.02 & $1.01-1.03$ \\
\hline \multicolumn{3}{|l|}{ Sex } \\
\hline Female $(n=463)$ & Ref & -- \\
\hline Male $(n=431)$ & 1.26 & $0.93-1.72$ \\
\hline \multicolumn{3}{|l|}{ Race/ethnicity } \\
\hline White $(n=278)$ & Ref & --- \\
\hline Black $(n=105)$ & 1.66 & $1.05-2.63$ \\
\hline Hispanic (n=306) & 1.28 & $0.87-1.88$ \\
\hline Unknown/other $(\mathrm{n}=205)$ & 0.79 & $0.49-1.26$ \\
\hline Charlson, per additional point & 1.09 & $1.04-1.14$ \\
\hline \multicolumn{3}{|l|}{ Hospitalization } \\
\hline \multicolumn{3}{|l|}{ C. difficile treatment } \\
\hline Metronidazole $(n=458)$ & Ref & --- \\
\hline Vancomycin $(n=70)$ & 1.11 & $0.64-1.93$ \\
\hline Both $(n=366)$ & 1.21 & $0.86-1.69$ \\
\hline \multicolumn{3}{|l|}{ Antibiotics } \\
\hline No $(n=241)$ & Ref & --- \\
\hline Yes $(n=653)$ & 1.35 & $0.91-2.00$ \\
\hline \multicolumn{3}{|l|}{ Immunosuppressants } \\
\hline No $(n=583)$ & Ref & --- \\
\hline Yes $(n=311)$ & 1.04 & $0.72-1.49$ \\
\hline \multicolumn{3}{|l|}{ ICU stay } \\
\hline No $(n=513)$ & Ref & --- \\
\hline Yes $(n=381)$ & 0.88 & $0.61-1.25$ \\
\hline Hospital LOS, per additional day $a$ & 1.00 & $1.00-1.01$ \\
\hline
\end{tabular}

Abbreviations: Ref, Reference; H2RA, histamine-2 receptor antagonist; ICU, intensive care unit; LOS, length of stay.

$a_{\mathrm{p}=0.14}$ 